American Journal of Applied Sciences 6 (7): 1359-1363, 2009

ISSN 1546-9239

(C) 2009 Science Publications

\title{
A Study of the Essential Oils of Four Sudanese Accessions of Basil (Ocimum basilicum L.) Against Anopheles Mosquito Larvae
}

\author{
${ }^{1}$ Azhari H. Nour, ${ }^{2}$ Salah A. Elhussein, ${ }^{2}$ Nour A. Osman, \\ ${ }^{3}$ Abduelrahman H. Nour and ${ }^{1}$ Mashitah M. Yusoff \\ ${ }^{1}$ Faculty of Industrial Sciences and Technology, University Malaysia Pahang, Kuantan, Malaysia \\ ${ }^{2}$ University of Gezira, Wad Medani, Sudan \\ ${ }^{3}$ Faculty of Chemical Engineering, University Malaysia Pahang, Kuantan, Malaysia
}

\begin{abstract}
Problem statement: Certain basil essential oils were claimed to have a larvicidal activity towards mosquito's larvae. To test this claim the essential oils of four accessions of basil grown in Sudan were selected and tested for Anopheles larvae. Malaria is the major health problem in the Sudan and the whole country is now considered endemic, with varying degrees, about 35,000 deaths every year due to malaria. Anopheles mosquito is the major vector of malaria disease in Sudan. Search for larvicidal active compound(s) is one of several attempts to fine effective and affordable ways to control this mosquito. To determine the toxic effects of basil essential oils extracted by steam distillation against Anopheles larvae. Approach: For the larvicidal bioassay, three concentrations (100, $300,500 \mathrm{ppm}$ ) of essential oil solutions of four basil accessions were prepared; $1 \mathrm{~mL}$ of ethanol was used to solubilize the oil in water $(999 \mathrm{~mL})$. In each concentration of oil solution were inserted 20 larvae (third instars). A set of controls using $0.1 \%$ ethanol and untreated sets of larvae in (tap) water, were also run for comparison. Data were evaluated through regression analysis, from the regression line; the $\mathrm{LC}_{50}$ values were read. The active ingredients were separated and/ or identified by TLC, IR and GC-MS. Results: Larvicidal activity of the essential oils is varied, lasted for about $9 \mathrm{~h}$ and thereafter decreased, $\mathrm{LC}_{50}$ values ranging from 190-300 ppm. Linalool, geraniol and eugenol are active components of basil essential oil against Anopheles larvae. Two accessions were caused 100\% mortality at a concentration range 300-500 ppm for $3 \mathrm{~h}$. Conclusion: These results indicated that basil essential oils have larvicidal activity towards Anopheles larvae. Therefore, could be affordable way to control this mosquito.
\end{abstract}

Key words: Basil, Ocimum basilicum, essential oil, larvicidal activity, Anopheles mosquito

\section{INTRODUCTION}

Mosquitoes are insects belonging to the order Diptera and as other true flies; develop through four distinct life stages-egg, larva, pupa and adult. It is hard to comprehend the amount of disease and the resulting sickness, death and economic loss caused by the mosquito ${ }^{[1]}$. Their attacks on farm animals can cause loss of weight and decreased milk production. Some mosquitoes are capable of transmitting diseases such as malaria, yellow fever and dengue. Malaria is transmitted by different Anopheles species, depending on the region and the environment ${ }^{[2]}$.

To control mosquito, any type of control should involve careful consideration of the biology of the mosquitoes and be based on scientific surveillance. A response to control nuisance mosquitoes may look very different from a response to control disease-vectoring mosquitoes $^{[3]}$. In all cases, larval mosquito control should be considered as the first option for abatement. This involves location of larval habitats, followed by their modification or treatment in such a way that the integrity of the habitat is preserved but the mosquito larvae are reduced in numbers ${ }^{[4]}$. By controlling larval mosquitoes, the adults may never become a problem. Larviciding has the greatest control impact on mosquito populations because the larvae are concentrated, immobile and accessible. Mosquitoes in the larval stage are attractive targets for pesticides because mosquitoes breed in water and thus, it is easy to deal with them in this habitat.

Corresponding Author: Azhari H. Nour, Faculty of Industrial Science and Technology, University Malaysia Pahang,

Tun Razak, 26300 Gambang. Kuantan, Pahang, Malaysia

Tel: +609-5492411 Fax: +609-5492766 H/P: +60179571471 
The use of conventional pesticides in the water sources, however, introduces many risks to people and/or the environment. Aromatic plants and their essential oils are very important sources of many compounds that are used in different respects. Essential oils and plant extracts are still an important natural resource and more promising for pesticides/ insecticides $^{[5]}$.

Therefore, the purpose of this study is to determine the activities of essential oils from four basil accessions (Ocimum basilicum L.) grown in Sudan were tested against third instars Anopheles mosquito larvae.

\section{MATERIALS AND METHODS}

Plant material (seed sources): Basil seeds used in these studies were obtained from different parts of Sudan. Commercial basil accession seeds were obtained from Germany and the United Arab Emirates (UAE). Seeds of basil accessions were directly sown on $60 \mathrm{~cm}$ wide ridges at the Demonstration Farm (Nishishiba), Fac. Agri. Sciences, University of Gezira, Wad Madani, Sudan. Sowing was done on Feb 20, 2005. Watering, weeding was carried out as necessary. No chemicals (fertilizers or others) were applied. Observations were made on growth and flowering of the plants weekly. Taxonomic identification of plants was performed by botanists of the National Centre for Research, department of biology, Khartoum, Sudan. The accessions were given numbers, as previously reported $^{[6]}$. Essential oils of 4 basil accessions obtained from fresh leaves of the plants by steam distillation were evaluated for larvicidal activity as described.

Rearing of mosquitoes larvae: The mosquitoes, Anopheles mosquito's larvae, were collected at various sites in Gezira state; the larvae were transferred separately in metal dishes $(40 \mathrm{~cm}$. diameter) and their rearing was continued until pupation. The larvae were reared continuously for several generations and kept under $25-30^{\circ} \mathrm{C}, \quad 70-80 \%$ relative humidity and photoperiod of 13:11 h (light/dark) on 4, August, 2006 to 15, September, 2006 in the insectary of Blue Nile Research and Training Institute, Wad Medani, Sudan. Larvae were fed on ground commercial biscuit. The adults were reared in humidified cages and supplied with $10 \%$ sugar solution and $10 \%$ multivitamin syrup supplied in plates. Female mosquitoes were periodically blood-fed on restrained rabbits to obtain protein used principally for egg production. Under these conditions, the full development from egg to adult lasted about 35 weeks. Third instars of Anopheles mosquito larvae were used in the larvicidal bioassay.

Larvicidal activity: For the larvicidal bioassay, the essential oil solutions of the four basil accessions were prepared; one $\mathrm{ml}$ of ethanol was used to solubilize the oil in water $(999 \mathrm{~mL})$. In $50 \mathrm{~m} \mathrm{~L}$ beakers containing $20 \mathrm{~mL}$ of each concentration of oil solution were inserted 20 larvae (third instars). With each experiment, a set of controls using $0.1 \%$ ethanol and untreated sets of larvae in (tap and distilled) water, were also run for comparison. Mortality was recorded after 1, 3, 6, 9, 12 and $24 \mathrm{~h}$, of test during which no nutritional supplement was added. The experiments were carried out at $30 \pm 1^{\circ} \mathrm{C}$. Each test comprised of three replicates with three concentrations $\left(100,300,500 \mu \mathrm{L} \mathrm{L}^{-1}\right)$. Data were evaluated through regression analysis. From the regression line; the $\mathrm{LC}_{50}$ values were read representing the lethal concentration for $50 \%$ larval mortality of Anopheles mosquito.

\section{RESULTS}

Larvicidal activity of the crude essential oils of four basil accessions: Table 1 shows larvicidal activity of essential oils of 4 basil accessions against Anopheles mosquito larvae. These accessions included basil grown in Sudan as ornamental plants (one accession, no. 10), one accession introduced from UA Emirates (no. 16), one accession introduced from Germany (no. 17) and accession no. 20 is grown in Sudan as wild basil.

Table 1: Larvicidal activity of essential oils of 4 basil accessions against mosquitoes (Anopheles) larvae

Percentage of mortality of larvae

\begin{tabular}{|c|c|c|c|c|c|c|c|c|c|c|c|c|c|c|c|c|c|c|c|c|c|c|c|c|c|c|c|c|}
\hline \multirow[b]{4}{*}{ Oi. } & \multicolumn{28}{|c|}{ Time of incubation } \\
\hline & 3 & & & & & & 6 & & & & & & 9 & & & & & & 12 & & & & & 24 & & & & \\
\hline & \multicolumn{28}{|l|}{ Tr. } \\
\hline & 10 & 16 & 17 & 20 & $\mathrm{Ci}$ & $\mathrm{Cii}$ & 10 & 16 & 17 & 20 & $\mathrm{Ci}$ & Cii & 10 & 16 & 17 & 20 & $\mathrm{Ci}$ & cii & 10 & 16 & 17 & 20 & $\mathrm{Ci}$ & Cii 10 & $16 \quad 17$ & 20 & $\mathrm{Ci}$ & Cii \\
\hline 100 & 10 & 15 & 20 & 0 & 0 & 0 & 40 & 30 & 37 & 0 & 0 & 0 & 40 & 35 & 37 & 10 & 0 & 0 & 40 & 35 & 37 & 10 & 0 & $\begin{array}{ll}0 & 40\end{array}$ & \begin{tabular}{|ll}
35 & 40 \\
\end{tabular} & 10 & 0 & 0 \\
\hline 300 & 40 & 75 & 60 & 55 & 0 & 0 & 50 & 85 & 95 & 55 & 0 & 0 & 60 & 95 & 95 & 75 & 0 & 0 & 60 & 95 & 95 & 75 & 0 & $0 \quad 60$ & $95 \quad 95$ & 75 & 0 & 0 \\
\hline 500 & 60 & 100 & 100 & 90 & 0 & 0 & 70 & 100 & 100 & 95 & 0 & 0 & 75 & 100 & 100 & 95 & 0 & 0 & 75 & 100 & 100 & 95 & 0 & $0 \quad 75$ & 100100 & 95 & 0 & 0 \\
\hline
\end{tabular}




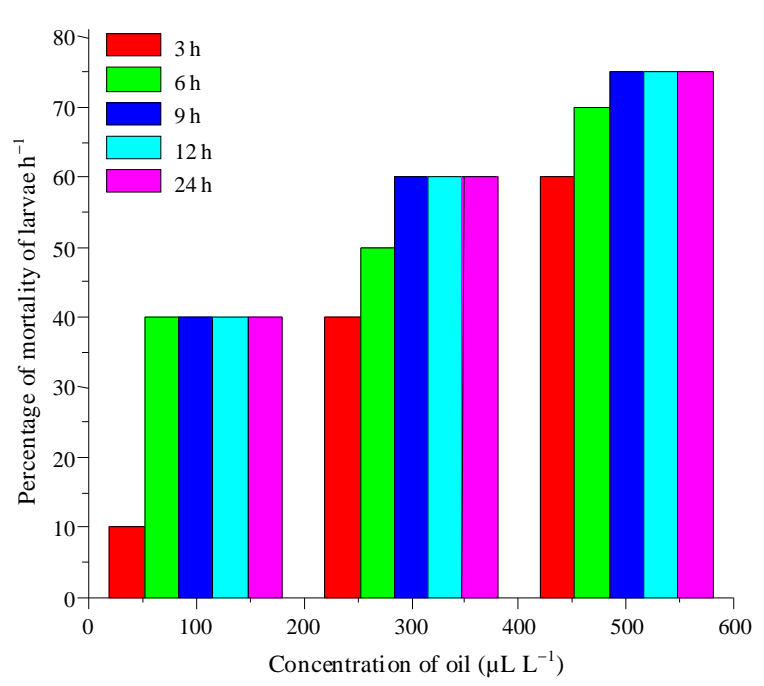

Fig. 1: Larvicidal activity of different concentrations of the essential oil of accession no. 10. (Measured at different time intervals (3-24 h) the results are expressed as \% mortality of mosquito larvae)

The oils were used in three concentrations and the \% mortality of the larvae was observed after 3, 6, 9, 12 and $24 \mathrm{~h}$ of incubation. All these essential oils of 4 accessions showed considerable larvicidal activity when tested against larvae of Anopheles mosquito.

The $\mathrm{LC}_{50}$ value (the lethal concentration for $50 \%$ of larval mortality of Anopheles larvae) was $190 \mu \mathrm{L} \mathrm{L}^{-1}$ for accession no. $16,200 \mu \mathrm{L} \mathrm{L}^{-1}$ for accession no. 17, $280 \mu \mathrm{L} \mathrm{L}^{-1}$ for accession no. 20 and $300 \mu \mathrm{L} \mathrm{L}^{-1}$ for accession no. 10. All essential oils of the 4 accessions were active larvicidally till $9 \mathrm{~h}$ of incubation. Thereafter, the activity remained constant between 9 and $12 \mathrm{~h}$ till $24 \mathrm{~h}$ of exposure of the larvae to the essential oils. Fig. 1 shows the Larvicidal activity of different concentrations of the essential oil of accession no. 10 measured at different time intervals (3-24 h) the results are expressed as \% mortality of mosquito larvae and Fig. 2a-c shows the mortality of larvae (\%) with different concentrations of oils $\left(\mathrm{A}=100 \mu \mathrm{L} \mathrm{L}^{-1}\right.$, $\mathrm{B}=300 \mu \mathrm{L} \mathrm{L}^{-1}$ and $\mathrm{C}=500 \mu \mathrm{L} \mathrm{L}^{-1}$ ).

The essential oil of accession no. 16 was subjected to Thin Layer Chromatography (TLC) analysis. Five TLC bands (corresponding zones detected using Anisaldehyde-sulfuric acid) were scraped off the plates, eluted and appropriately tested for larvicidal activity.

Three bands were found larvicidally very active, $100 \%$, mortality reached within just half-an-hour of exposure (the concentration was not accounted for, roughly equivalent to $50 \mathrm{mg}$ of crude essential oil). The remaining two bands were inactive.

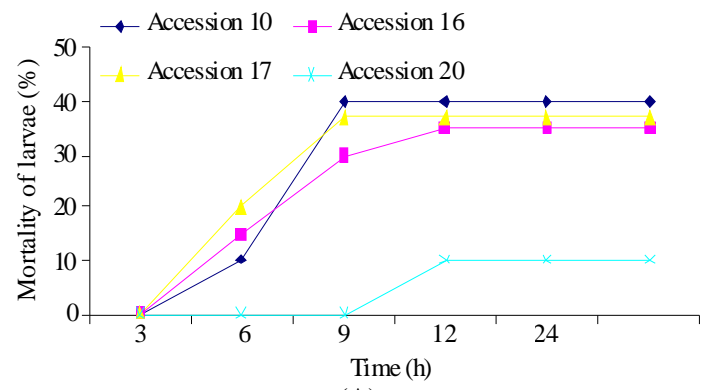

(A)

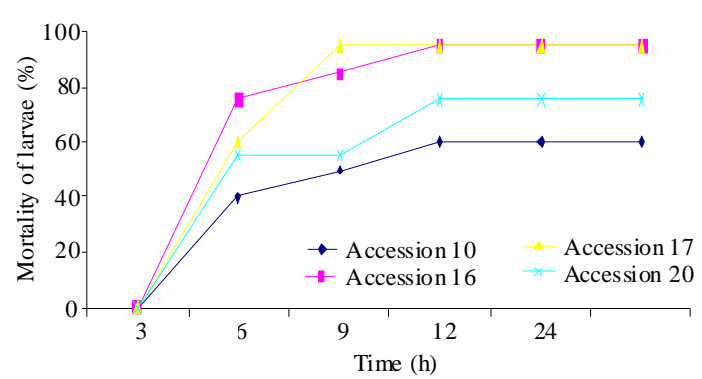

(B)

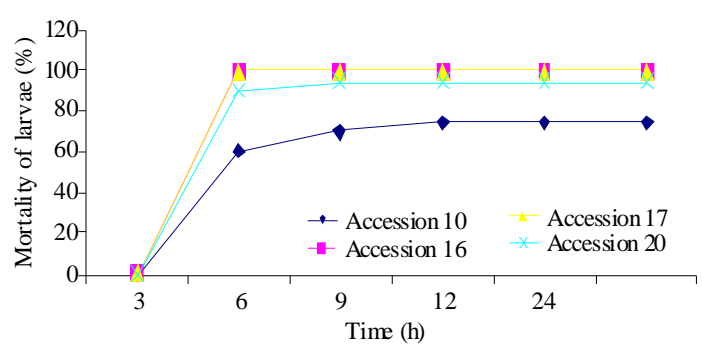

(C)

Fig. 2A-C: Mortality of larvae (\%) with difference concentrations of oils $\left(\mathrm{A}=100 \mu \mathrm{L} \mathrm{L}^{-1}\right.$, $\mathrm{B}=300 \mu \mathrm{L} \mathrm{L}^{-1}$ and $\left.\mathrm{C}=500 \mu \mathrm{L} \mathrm{L}^{-1}\right)$

TLC elutes of the three larvicidally active compounds were subjected to i.r. spectrum analysis (in chloroform solution). We studied the i.r. spectra, absorption frequencies observed and the possible functional groups present in the three bands (compounds) as interpreted from the literature ${ }^{[7]}$. These data suggest that the active bands (compounds) are eugenol, geraniol and linalool.

\section{DISCUSSION}

Larval mortality increased as the essential oil concentration increased. The essential oils of the different accessions had differential activity. After $3 \mathrm{~h}$ of treatment, for example, the highest dose $\left(500 \mu \mathrm{L} \mathrm{L}^{-1}\right)$ caused $100 \%$ mortality in the case of accessions (no. 16) and (no. 17), 90\% and 60\% mortality with 
accessions (no. 20) and (no. 10) respectively. This order of larvicidal potency (16 and $17>20>10)$ remained the same for all treatment periods and concentrations except for the anomaly of accession no. 20 having exceptionally little activity at the lower concentration.

In previous study, authors reported larvicidal activity of essential oil against Aedes aegypti at concentration of $(2,4,6,8$ and $10 \%)$. Activity was different for the different instars stages ${ }^{[8]}$. However, these authors used a concentration much higher than the one used in this study (the concentration used here was $0.01 \%)$. In another study author recorded different mortalities for larvicidal activity of Ocimum sanctum leaf extraction with acetone, chloroform, ethyl acetate, hexane and methanol against Aedes aegypti larvae ${ }^{[9]}$. Previous studies reported that some basil species showed solvent extracts from the whole plant have larvicidal properties against Anopheles mosquitoes' larvae $\mathrm{e}^{[10-12]}$.

The i.r. suggestion of three bands of accession no. 16 shown eugenol, geraniol and linalool are active compounds. It is noteworthy that the major components of the essential oil of accession no. 16 as determined by GLC were methyl cinnamate $(40.42 \%)$, linalool $(29.43 \%)$, cineole $(9.48 \%)$, thujone $(9.01 \%)$, methyl chavicol $(6.37 \%)$ and eugenol $(1.24 \%)$, as previously reported $^{[6]}$. Thus Linalool, a major component, is likely to be one of the active compounds. This is supported by reported literature ${ }^{[13]}$. The two other larvicidally active compounds are eugenol, geraniol, the structure of which was suggested by i.r. spectra and are present as minor components in accession 16 .

In this study, we tested the larvicidal activity of four accessions of basil essential oils grown in Sudan against Anopheles mosquito larvae. The results obtained from this study indicate that all four essential oils of basil should promising larvicidal activity against Anopheles mosquito larvae. These results, somewhat are same with some previous studies in larvicidal activity of basil essential oil against mosquito larvae; but there also some differ with some reported studies, the differences can be attributed to many factors; perhaps the most obvious is the difference in concentration or amount of essential oil and oil obtaining method (some authors obtained the oil by solvent extraction, while for this study the essential oils obtained by steam distillation). However, comparisons, such as the preceding, are confounded by differences in the mosquito species larvae tested, basil species and environmental factors. So, further investigations are needed to elucidate the four essential oils against a wide range of mosquito species larvae and so far the formulation of active ingredients.

\section{CONCLUSSION}

In conclusion, the essential oils of Ocimum spp., namely accessions no. 16 and no. 17 were shown to be quite potent as larvicidal agents against Anopheles mosquito larvae and caused $100 \%$ mortality at a concentration range of $300-500 \mu \mathrm{L} \mathrm{L}^{-1}$ for $3 \mathrm{~h}$ and caused $95 \%$ mortality at a concentration of $300 \mu \mathrm{L} \mathrm{L}^{-1}$ for $6 \mathrm{~h}$. These results are very promising in creating new effective and affordable approaches to the control of Anopheles mosquito and larvae.

\section{ACKNOWLEDEMENT}

The researchers must acknowledge partial financial (grant No. MHESR, SR, 1, A, 13, 1-2005) support offered by the "Basil Project" at NOPRI, University of Gezira. This project is a kind research grant from the Ministry of Higher Education and Scientific Research, Khartoum. We also thank members of Blue Nile Research and Training Institute, Wad Medani, Sudan for their cooperation, made allowance and using their lab and insectary for this study.

\section{REFERENCES}

1. Burgess, N., 1993. Colored Atlas of Medical Entomology. Chapman and Hall Medical, London, 1st Edn., pp: 1-6. ISBN: 10: 0412323400, pp: 144.

2. Burfield, T. and S. Reekie, 2005. Mosquitoes, malaria and essential oils. Int. J. Aromather., 15: 30-41. DOI: 10.1016/j.ijat.2004.09.013

3. Lee, S.E., 2000. Mosquito larvicidal activity of piper nonaline, piperidine alkaloid derived from long pepper, piper longum. J. Am. Mosq. Control Assoc., 16: 245-247. http://cat.inist.fr/?aModele $=$ afficheN\&cpsidt $=816264$

4. Cavalcanti, E., S. Morais, M. Alima and E. Santana, 2004. Larvicidal activity of essential oils from Brazilian plants against Aedes Aegyptil. J. Oswaldo Cruz, Rio de Janeiro, 99: 541-544. DOI: 10.1590/S0074-02762004000500015

5. Amer, A. and H. Mehlhorn, 2006. Larvicidal effects of various essential oils against Aedes, Anopheles and Culex larvae (Diptera, Culicidae), Parasitol. Res., 99: 466-472. DOI: 10.1007/s00436-006-0182-3

6. Abduelrahman, A., S. Elhussein, N. Osman and A. Nour, 2009. Morphological variability and chemical composition of essential oils from nineteen varieties of basil (Ocimum basilicum L.) growing in Sudan. Int. J. Chem. Technol., 1: 1-10. DOI: $10.3923 /$ ijct.2009.1.10 
7. Fessenden, R. and J. Fessenden, 1979. Organic Chemistry. 2nd Edn., Willard Grant Press Boston, Masscachusetts.

http://www.ecampus.com/book/9780871507242

8. Murugan, K., P. Murugan and A. Noortheen, 2007. Larvicidal and repellent potential of Albizzia amara Boivin and Ocimum basilicum Linn against dengue vector, Aedes aegypti. Biores. Technol., 98: 198-201.

http://cat.inist.fr/?aModele=afficheN\&cpsidt=18457172

9. Anees, A., 2008. Larvicidal activity of Ocimum sanctum Linn. (Labiatae) against Aedes aegypti (L.) and Culex quinquefasciatus (Say). J. Parasitol. Res., 103: 1451-1453. DOI: 10.1007/s00436-008-0991-7

10. Cheng, S., H. Chang, S. Chang, K. Tsai and W. Chen, 2003. Bioactivity of selected plant essential oils against the yellow fever mosquito Aedes aegypti larvae. Biores. Technol., 89: 99-102. DOI: 10.1016/S0960-8524(03)00008-7
11. Keita, S., V. Charles, P. Jeen, T. John and B. Andre, 2001. Efficacy of essential oil of Ocimum basilicum L. and $O$. gratissimum L. applied as an insecticidal fumigant and powder to control Callosobruchus Maculates (fab.) J. Stored Prod. Res., 37: 339-349. http://cat.inist.fr/?aModele $=$ afficheN\&cpsidt=14101781

12. Rajkumar, S. and A. Jebanesan, 2008. Bioactivity of flavonoid compounds from Poncirus trifoliate L. (Family: Rutaceae) against the dengue vector, Aedes aegypti L. (Dipteria: Culicidae). J. Parasitol. Res., 104: 19-25. DOI: 10.1007/s00436-008-1145-7

13. Laurent, D., A. Vilaseca, J. Chantraine, C. Ballivian, G. Saavedra and R. Ibanez, 1997. Insecticidal activity of essential oils on Triatoma infestans. Phytother. Res., 11: 285-290. DOI: 10.1002/(SICI)1099-1573(199706)11:4<285 